Neurologist. 2012 July ; 18(4): 229-232. doi:10.1097/NRL.0b013e31825bbf4d.

\title{
An Unusual Case of Stroke
}

\author{
Michael Rosario, MD, \\ Department of Neurology, University of Connecticut, Hartforf Hospital Hartford, Connecticut
}

Xianyuan Song, MD, PhD, and

Department of Pathology, Hartford Hospital, Hartford, Connecticut

Louise D. McCullough, MD, PhD.

Department of Neurology, University of Connecticut Health Center and The Stroke Center at Hartford Hospital, 263 Farmington Ave, Farmington CT, 06030, Phone (860) 679-3186; Fax (860) 679-1181

Louise D. McCullough: Imccullough@uchc.edu

\section{Keywords}

Stroke; Vasculitis; Cryptococcus; MRI

\section{Introduction}

A clinical presentation involving fluctuating symptoms, multiple strokes, cortical symptoms, confusion, severe headache, and diffuse central nervous system involvement with involvement of both the white matter and gray matter, as well as supratentorial and infratentorial brain regions, suggests CNS vasculitis. Many other conditions may mimic vasculitis, especially since there is no the presentation of CNS vasculitis can be so varied. New imaging techniques have allowed for the rapid and accurate diagnosis of stroke. In this case we present a patient with multifocal ischemia on MRI initially thought to represent a rapidly progressive CNS vasculitis.

Case

A 58-year-old female presented with a 2 week history of lethargy, fatigue, and progressive gait instability. A non-enhanced CT of the head was normal from an outside Emergency Department (ED) 1 week prior to admission. Three days prior to transfer to our hospital, she returned to the outside hospital $(\mathrm{OSH})$ after presenting with headache, inattentiveness, confusion, nausea, and generalized weakness. A repeat head CT scan showed a subacute infarction in the right caudate and an acute infarction of the right putamen. She was admitted and a MRI of the brain showed acute bilateral cerebellar and right basal ganglia infarcts with a normal MRA (Figure 1a). She was transferred for further stroke work up.

The patient had multiple vascular risk factors including a history of poorly controlled hypertension, hyperlipidemia, Non-insulin dependent diabetes mellitus and obesity (BMI: 42). She also had a history of hypothyroidism, auto-immune non-alcoholic liver cirrhosis diagnosed three years prior to admission, osteoarthritis, and chronic back pain. Her home medications included levothyroxine, furosemide, lisinopril and insulin. Several days after admission, family members noted that the patient had been non-compliant with her insulin,

Correspondence to: Louise D. McCullough, lmccullough @uchc . edu.

There are no conflicts to disclose 
thyroid and anti-hypertensive medications for several months. She had also been taking prednisone $5 \mathrm{mg}$ daily and occasional narcotics for a diagnosis of polymyalgia rheumatica and chronic back pain. She was a smoker, consumed alcohol occasionally, and denied any recreational drug abuse. Family history was significant for early heart disease in both her father and brother.

On initial ED exam the BP was 162/94 mm Hg; she was afebrile. She was awake but inattentive, requiring multiple verbal cues and frequent redirection to maintain eyes open. Her best verbal response was inappropriate words. She was not reliably following simple commands. No papilledema was observed. Cranial nerve examination revealed a left gaze preference, rotatory nystagmus in both directions on horizontal gaze, a flattened right nasolabial fold and a poor gag reflex. Corneal and oculocephalic reflexes were intact. She did not move her extremities spontaneously but withdrew and localized to pain in all four extremities as best motor response. Reflexes were symmetrical with bilateral plantar flexor responses. Coordination could not be tested. The patient underwent endotracheal intubation for airway protection. The Glasgow Coma Score (GCS) on admission was 12.

Initial laboratory testing (Table 1) showed a urinary tract infection. Other notable findings included significant hyperlipidemia and a high TSH consistent with her history of poor medication compliance.

On Day \#2 a brain MRI with contrast (Figure 1b) was performed (3 days after the OSH MRI). This demonstrated new bilateral anterior cerebral artery (ACA) infarctions, and a new left temporoparietal infarction. There was no abnormal enhancement. Subsequent transthoracic echocardiogram showed normal ventricular systolic function, normal valvular anatomy, and the presence of a patent foramen ovale without an atrial septal aneurysm. Transesophageal echocardiogram was deferred secondary to the presence of esophageal varicies. There was no evidence of deep vein thrombosis on lower extremity ultrasound. CT angiogram of the head did not show any focal stenosis or aneurysm (Figure 2a). CT angiogram of the neck showed normal extracranial vasculature.

At this point autoimmune vasculopathies, primary CNS angiitis and intravascular large -Bcell lymphoma were considered due to her fluctuating symptoms, cortical strokes, confusion and headache. A lumbar puncture (LP) was attempted on hospital day 2 but due to body habitus, the initial attempt was unsuccessful. She underwent fluoroscopic-guided LP on hospital Day 3 (Table 1).

The patient was started on acyclovir for possible herpetic infection and Dexamethasone $1 \mathrm{~g}$ IV daily due to concerns of vasculitis. Rheumatologic work up demonstrated the presence of elevated thyroid peroxidase ( 51 , normal $<35 \mathrm{IU} / \mathrm{mL}$ ) and Thyroglobulin $(>4,000$, normal $<115 \mathrm{IU} / \mathrm{mL}$ ) antibodies suggestive of Hashimoto's Thyroiditis, supporting the possibility of an autoimmune vasculitis. Cerebral angiogram (Day 3) showed no evidence of vasculitis (Figure 2b). On Day 4 a brain and meningeal biopsy was performed. The pathology was consistent with "an organized infarct without presence of vasculitis". Further testing included CT scan of thorax, abdomen, and pelvis which showed evidence of cirrhosis and portal hypertension, with no evidence of malignancy or emboli.

Five days after admission, CSF culture and India Ink preparation were positive for Cryptococcus neoformans. Review of the brain biopsy show the presence of budding yeast in the meninges (Figure 1c and 1d). The patient was started on amphotericin B and flucytosine. The patient's clinical condition continued to deteriorate and the patient subsequently passed away on hospital day \# 11, from septic shock and multi-organ failure secondary to antifungal therapy. 


\section{Discussion}

Cryptococcal meningoencephalitis (CM) is an important opportunistic infection in patients with a compromised cellular immune response, particularly those with HIV infection. Among non-HIV-infected patients with CM, the majority are immunocompromised. Patients treated with glucocorticoids or other immunosuppressive therapies or chronic organ failure such as liver, lung, and kidney failure also have a higher incidence. ${ }^{1}$ Non-HIV patients with $\mathrm{CM}$ often develop lethargy, coma, malaise, personality changes, and memory loss rather than fever and headache and therefore the diagnosis is often delayed. ${ }^{1,2,3}$ This patient presented with atypical multiple, bilateral, large vessel strokes. Although vasculitis can occur with CM, if infarcts are seen, they are primarily deep small vessel events in the basal ganglia, internal capsule, and thalamus. ${ }^{2,3,4}$ Multiple large vessel cortical infarctions have not been previously reported with CM. Several mechanisms may contribute to the development of infarctions in CM. As CM often leads to a predominantly basilar meningitis, vessels that transverse the base of the brain can become covered with exudate and develop vasculitis with inflammation, vasospasm, constriction, and eventually thrombosis. The largest retrospective study to date identified 28 patients with cerebral infarctions secondary to chronic meningitis; $60 \%$ of these were secondary to tuberculous meningitis and $39 \%$ had $\mathrm{CM}$. Among the patients with CM, most involved the basal ganglia or internal capsule; however multiple infarcts were seen in almost $50 \% .^{2}$ Interestingly, no infarcts were seen in the cerebellum or in the MCA territory in that case series, both of which were seen in this patient, obscuring the diagnosis.

This case was particularly challenging as this patient had a variety of underlying vascular risk factors that predisposed her to vessel thrombosis, she had been transferred with the diagnosis of "multiple strokes", leptomeningeal enhancement was absent, she was afebrile on admission, and the family was unaware of her chronic prednisone use. The rapid progression of multiple, bilateral, cortical and subcortical strokes led our original diagnostic work up towards CNS vasculitis. In CNS vasculitis, headache and encephalopathy are the most frequent initial symptoms, consistent with her presentation. Symptoms or signs of vasculitis outside of the central nervous system are rare; serologic markers of inflammation are typically normal. MRI is abnormal in more than $90 \%$ of patients, but the pattern of findings is not specific. CSF analysis often reveals modest, nonspecific elevation in protein or mild pleocytosis. ${ }^{5}$ Angiography has a relatively low sensitivity for detecting CNS vasculitis ${ }^{5}$ and brain biopsy is often used to confirm the diagnosis, however even the sensitivity of brain biopsy may be quite low. ${ }^{6}$

The presence of thyroid peroxidase and thyroglobulin antibodies also led us to consider Hashimoto Encephalitis (HE). This disease often presents with cognitive impairment or seizures, but stroke-like events and focal neurological deficits can occur. ${ }^{7}{ }^{8}$ In retrospect, the initial LP findings on day 3 were unusual for PACNS, as the CSF pleocytosis was much higher than that usually seen in CNS vasculitis. However, a rapidly progressive variant of PACNS has been recently described in which patients present with severe neurological impairment, some with CSF WBC counts $>400$ per $\mathrm{mm}^{3}$. These patients tend to have bilateral involvement of large vessels and a rapidly progressive course manifested by multiple recurrent cerebral infarctions as in this case. ${ }^{9} \mathrm{HSV}$ was considered, and acyclovir was added, but CM was only considered when the biopsy and CSF results came back positive for Cryptococcus. This patient was immune compromised from long standing liver disease as well as chronic corticosteroid use, which was unknown at the time of her presentation.

In summary, cerebral infarction is rare in CM. When it does occur, the vast majority have small vessel involvement. Stroke involving the large vessels is very rare, and no previous 
reports have documented the extensive, multiple, cortical infarctions that were seen in this patient. Opportunistic CNS infections are important to consider in the differential diagnosis of any patient, especially when clues exist that suggest the patient is immunocompromised. This is the first report of multiple large vessel strokes secondary to cryptococcal meningitis and emphasizes the importance of considering fungal infections in the differential diagnosis of stroke.

\section{References}

1. Bicanic T, Harrison T. Cryptococcal meningitis. British Medical Bulletin. 2004; 72:99-118. [PubMed: 15838017]

2. Lan SH, Chang WN, Lu CH, Lui CC, Chang HW. Cerebral infarction in chronic meningitis: a comparison of tuberculous meningitis and cryptococcal meningitis. QJM. 2001; 94:247-253. [PubMed: 11353098]

3. Saul RF, Gallager JG. Sudden hemiparesis as the presenting sign in cryptococcal meningoencephalitis. Stroke. 1986; 17:753-754. [PubMed: 3738961]

4. Aharon-Peretz JJ, Kliot D, Finkelstein R, et al. Cryptococcal meningitis mimicking vascular dementia. Neurology. 2004; 62:2135. [PubMed: 15184639]

5. Salvarani C, Brown RD Jr, Calamia KT, et al. Primary central nervous system vasculitis: analysis of 101 patients. Ann Neurol. 2007; 62:442-451. [PubMed: 17924545]

6. Birnbaum J, Hellmann D. Primary Angiitis of the Central Nervous System. Archives of Neurology. 2009; 66:704-709. [PubMed: 19506130]

7. Mijajlovic M, Mirkovic M, Dackovic J, et al. Clinical manifestations, diagnostic criteria and therapy of Hashimoto's encephalopathy: Report of two cases. Journal of the Neurological Sciences. 2010; 288:194-196. [PubMed: 19846119]

8. Bonmann E, Prumbaum M, Stippich C, et al. Hashimoto Encephalopathy Rare Cause of Stroke in Young Patient. The Neurologist. 2009; 15:342-344. [PubMed: 19901715]

9. Salvarani C, Brown RD Jr, Calamia KT, et al. Rapidly progressive primary central nervous system vasculitis. Rhuemathology. 2011; 50(2):349-58. 

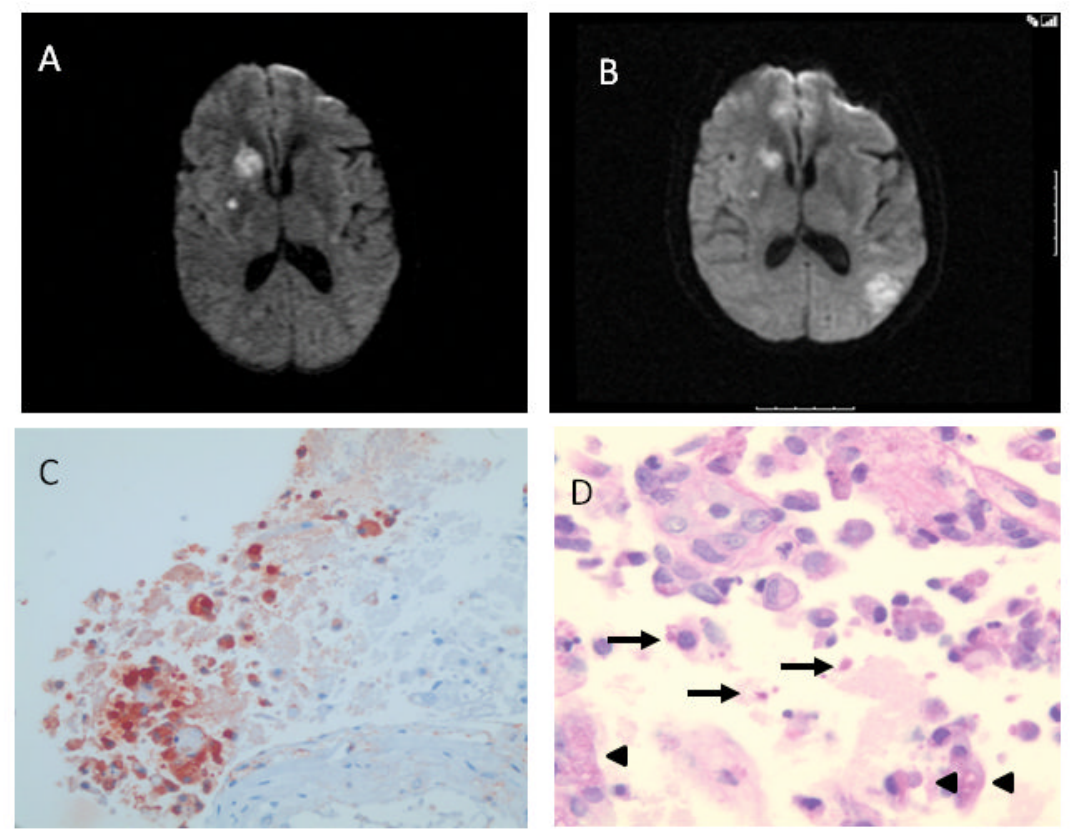

Figure 1.

A: Diffusion weighted MRI (DWI) at an outside hospital 3 days prior to admission showing an acute infarct in the right basal ganglia and right caudate. B: DWI on admission showing a new infarct in the left temporal parietal area and increased diffusion in the anterior cerebral artery territory bilaterally. Hemispheric cerebellar infarcts were also present (not shown). C: Immunohistochemical staining for Cryptococcus in the brain biopsy specimen is strongly positive (400X). D: High magnification of the brain biopsy specimen shows budding yeast in both the extracellular compartment and within macrophages (arrow points to a budding yeast; arrowhead shows an intracellular yeast (PAS stain at 1000x). 

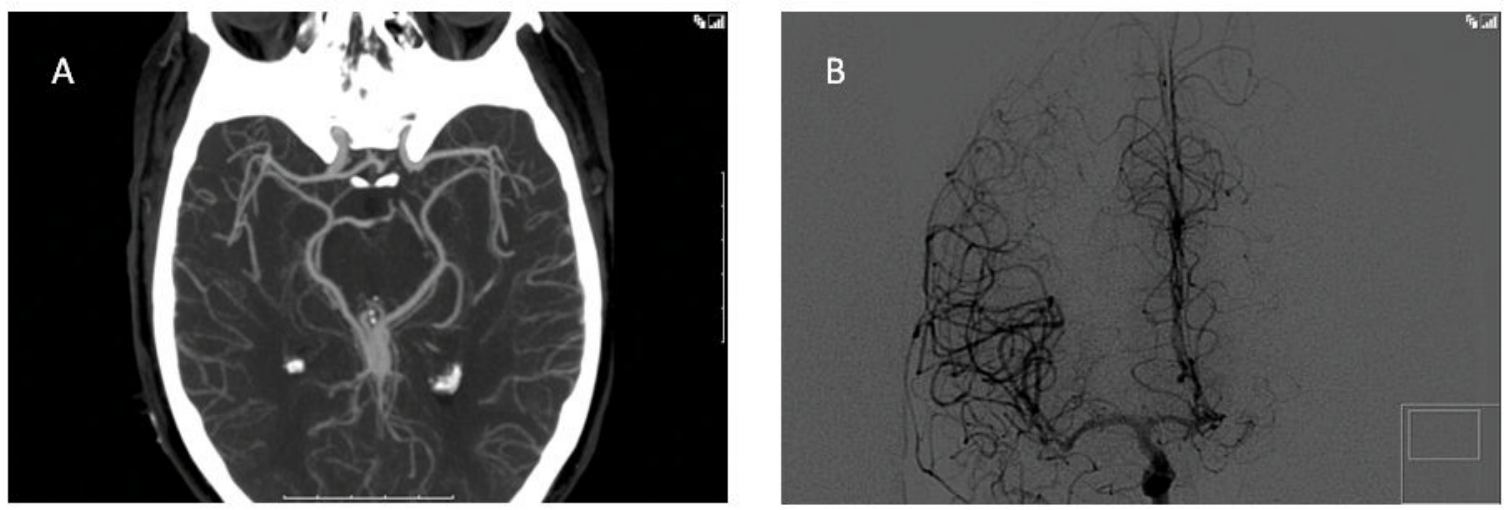

Figure 2.

A: Normal CT Angiogram of intracranial vasculature. B: Conventional angiogram lacking angiographic evidence of vasculitis. 


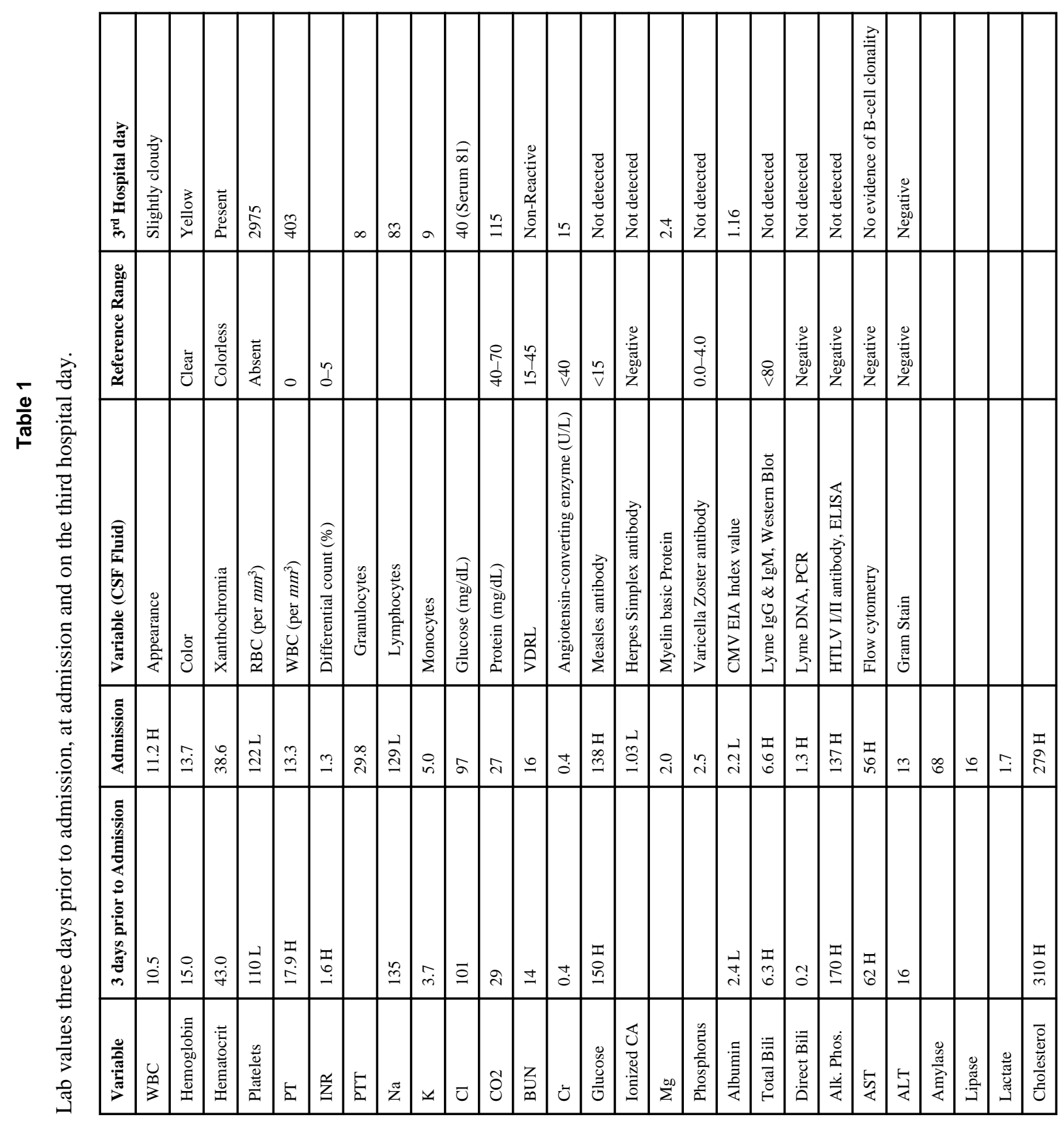




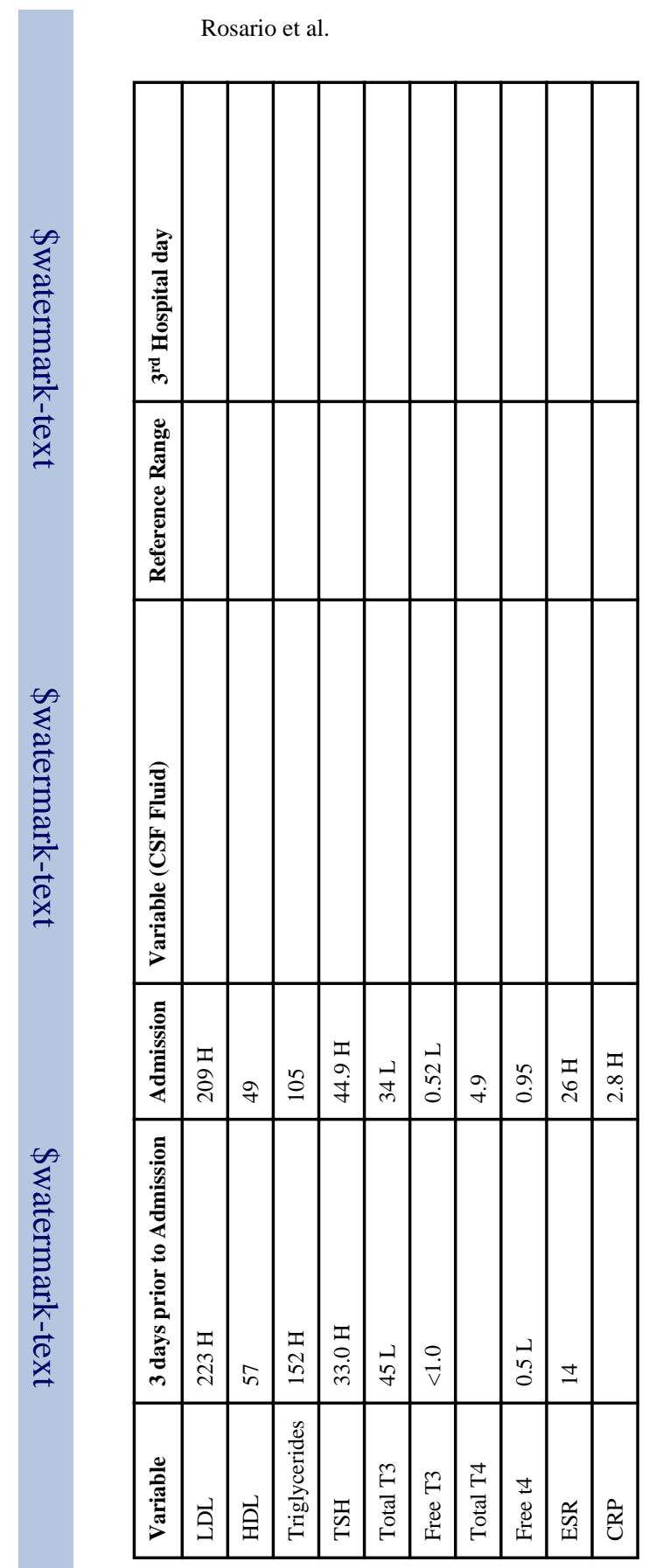

Neurologist. Author manuscript; available in PMC 2013 July 01. 